\title{
Accompagner les infırmières et les étudiantes dans la réflexion sur des situations de soins : Un modèle pour les formateurs en soins infirmiers
}

\author{
Patrick Lavoie \\ Université de Montréal, patrick.lavoie.1@umontreal.ca \\ Louise Boyer \\ University of Montreal, louise.boyer@umontreal.ca \\ Jacinthe I. Pepin \\ Université de Montréal, jacinthe.pepin@umontreal.ca \\ Johanne Goudreau \\ University of Montreal, johanne.goudreau@umontreal.ca \\ Odile Fima \\ AFORCES, Le Mans, France, odile.fima@gmail.com
}

Follow this and additional works at: https://qane-afi.casn.ca/journal

Part of the Educational Methods Commons, and the Nursing Commons

\section{Recommended Citation}

Lavoie, Patrick; Boyer, Louise; Pepin, Jacinthe I.; Goudreau, Johanne; and Fima, Odile (2017) "Accompagner les infirmières et les étudiantes dans la réflexion sur des situations de soins : Un modèle pour les formateurs en soins infirmiers," Quality Advancement in Nursing Education - Avancées en formation infirmière: Vol. 3: Iss. 1, Article 3. DOI: https://doi.org/10.17483/2368-6669.1100

This Article is brought to you for free and open access by Quality Advancement in Nursing Education - Avancées en formation infirmière. It has been accepted for inclusion in Quality Advancement in Nursing Education - Avancées en formation infirmière by an authorized editor of Quality Advancement in Nursing Education - Avancées en formation infirmière. 


\section{Accompagner les infirmières et les étudiantes dans la réflexion sur des situations de soins : Un modèle pour les formateurs en soins infirmiers}

\section{Cover Page Footnote}

Les travaux cités ont été rendus possibles par des bourses doctorales du Réseau de recherche en interventions en sciences infirmières du Québec (soutenue par les Fonds de recherche du Québec (FRQ) Santé), des FRQ - Société et culture, de l'Équipe FUTUR (soutenue par le FRQ-Société et culture), du Ministère de l'enseignement supérieur, de la recherche et du sport du Québec, de la Faculté des sciences infirmières de Université de Montréal et de la Fondation Marc-Chouinard. Les études présentées ont également été soutenues par la Fondation canadienne pour la recherche sur les services de santé. The cited works were achieved thanks to doctoral awards from the Réseau de recherche en interventions en sciences infirmières du Québec (supported by the FRQ [Fonds de recherche du Québec] Health division), the FRQ Social and cultural division, the FUTUR team (supported by the FRQ Social and cultural division), the Québec's Ministère de l'enseignement supérieur, de la recherche et du sport, the Faculty of Nursing at Université de Montréal and the Marc-Chouinard Foundation. The studies in question were also supported by the Canadian Foundation for Healthcare Improvement. 


\section{Introduction}

Dans la francophonie, la dernière décennie a été marquée par une mouvance des programmes de formation infirmière vers une approche par compétences. En plus d'une orientation explicite vers l'atteinte de résultats d'apprentissages, cette approche met en avant l'apprentissage par des stratégies pédagogiques actives (Frank et al., 2010; Goudreau et al., 2009). Celles-ci sont dites actives en raison de la volonté de mobiliser les apprenants dans des situations proches de leur future pratique professionnelle, tout en les incitant à réfléchir sur leurs processus d'apprentissage (Bonwell \& Eison, 1991; Prince, 2004). La simulation clinique, l'apprentissage par problème ou les stages en milieu de pratique en sont des exemples parlants ; les apprenants y sont confrontés à des situations cliniques réelles ou réalistes comme occasions d'apprentissage.

Ceci soulève des questions quant au rôle des formateurs dans l'accompagnement des apprenants pour maximiser l'apprentissage au moyen de stratégies pédagogiques actives. Il s'agit d'une pratique qui combine à la fois l'expertise clinique des formateurs, mais aussi leur connaissance et leur expérience de démarches réflexives. Les formateurs doivent s'approprier des modalités d'accompagnement fondées sur des connaissances théoriques et empiriques issues des sciences infirmières et des sciences de l'éducation.

C'est dans cette logique qu'un modèle de réflexion sur des situations cliniques a été développé et mis à l'essai par une équipe de chercheurs universitaires québécois. Ce modèle vise plus particulièrement l'apprentissage du raisonnement et du jugement cliniques infirmiers, compétences centrales à l'exercice infirmier. Dans cet article à visée didactique, le modèle et ses fondements théoriques sont détaillés de même que des résultats de recherche quant à sa mise en œuvre dans différentes stratégies pédagogiques actives.

\section{Fondements théoriques}

\section{Jugement clinique infirmier}

Le concept de jugement clinique infirmier s'inscrit dans le courant de recherches sur la prise de décision infirmière. Ces recherches se sont inscrites dans trois principales perspectives théoriques : la théorie du traitement de l'information, la théorie de l'analyse décisionnelle et la théorie herméneutique, c'est-à-dire la science de l'interprétation et de la compréhension du sens (Banning, 2008; Higgs, 2008; Tanner, 2006). De ces différentes approches ont émergé un vaste éventail de connaissances, que Tanner (2006) synthétise dans une conceptualisation du processus décisionnel infirmier, qu'elle nomme « Modèle du jugement clinique en soins infirmiers ». Dans ce modèle, le jugement clinique est défini comme «une interprétation ou une conclusion à propos des besoins, inquiétudes ou problèmes de santé d'un patient et la décision d'entreprendre ou non une action (...) selon ce qui semble approprié en vertu de la réponse du patient » [traduction libre] (Tanner, 2006, p. 204). Le raisonnement clinique y est défini comme le processus par lequel l'infirmière arrive à poser un jugement clinique.

Le jugement clinique correspond à la compréhension qu'une infirmière se forge de la situation de santé d'un patient, en vertu de leur interaction. Cette interaction peut être conçue sous l'angle de la démarche clinique, par laquelle l'infirmière recueille, analyse et interprète des données pour planifier ses interventions, les conduire et en évaluer les résultats (Alligood, 2013). L'infirmière prend part à cette interaction avec tout son bagage professionnel et personnel. Elle a acquis des connaissances théoriques lors de sa formation, ainsi que d'autres connaissances au fil 
de son expérience professionnelle auprès de certaines clientèles. Sur le plan personnel, ses valeurs, ses représentations et ses repères moraux ou éthiques sont autant de facteurs qui influenceront son approche de la situation d'un patient. Qui plus est, le contexte dans lequel elle pose son jugement clinique serait influant, tant par les structures politiques et sociales en place que par la culture du milieu clinique, les routines et les manières de faire qui sont plus ou moins ancrées dans les pratiques de l'infirmière.

Tous ces facteurs se combinent dans la représentation que s'est construite l'infirmière de ce qui devrait se présenter à elle, lorsqu'elle ira à la rencontre du patient. Ses attentes face à la situation de santé du patient sont le produit de son bagage professionnel et personnel, mais aussi de la connaissance qu'elle a développée de ce patient unique et de ses réactions aux soins. Dans l'interaction, l'infirmière confronte ses attentes à ce qui se présente et remarque des écarts lorsque ses représentations ne correspondent pas à ce qu'elle observe chez le patient. Remarquer, ou saisir la situation, constitue la première étape du processus qui la mènera à poser un jugement clinique.

L'infirmière interprètera les données remarquées selon différents modes de raisonnement. L'utilisation d'un mode de raisonnement en particulier dépend de plusieurs facteurs, dont l'expertise de l'infirmière. Des résultats de recherche indiquent que l'infirmière novice scrute les données selon un mode analytique, décortiquant chaque élément de la situation afin d'établir des hypothèses et d'en vérifier la plausibilité (Banning, 2008; Boyer, Tardif, \& Lefebvre, 2015; Higgs, 2008; Tanner, 2006). L'experte, quant à elle, peut saisir instantanément la nature de la problématique qui se présente à elle et savoir d'emblée quelles seraient les actions à entreprendre (Benner, 1984; Benner, Tanner, \& Chesla, 2009). Ce mode intuitif résulte d'une intégration avancée de différentes situations de soins, qui fait que des ensembles de données constituent des portraits cliniques connus et reconnaissables. Il est à noter que les infirmières peuvent utiliser une grande variété de stratégies cognitives lors de l'exploration de situations cliniques, les recherches de Fonteyn (1998) ayant permis d'en identifier plus de 70 différentes.

Au terme de cette démarche d'interprétation des données remarquées, l'infirmière arrivera à une conclusion, soit son jugement clinique. Elle décidera de la conduite à tenir, des actions à mettre en place et amorcera ses interventions. Au fil de ses interventions, elle s'ajuste en tenant compte de la réaction du patient et des différents éléments de son environnement. C'est ainsi que Tanner (2006) intègre la notion de réflexion dans l'action pour désigner cette sensibilité à la réponse du patient et à l'évolution de la situation.

Une fois que l'interaction est terminée et que l'issue en est connue, l'infirmière pourra prendre le temps de réfléchir sur l'action. La réflexion, concept tiré des théories de l'apprentissage expérientiel (Dewey, 1997, 2007), constitue la clé de voûte du développement du jugement clinique infirmier. Ce serait cette démarche de retour sur l'expérience qui permettrait à l'infirmière d'apprendre et de réinvestir ses apprentissages dans sa pratique future.

\section{Apprentissage expérientiel et réflexion}

L'accent sur le concept de réflexion dans le modèle du jugement clinique de Tanner (2006) invite à se pencher sur les théories de l'apprentissage expérientiel, plus particulièrement sur les écrits de Dewey $(1997,2007)$ à qui Tanner (2006) attribue l'origine du concept de réflexion. Dans la philosophie de Dewey (1997), l'expérience est une occasion d'apprentissages qui, à leur tour, seront réinvestis dans de futures expériences. L'expérience est définie comme une transaction entre un organisme (l'infirmière) et son environnement (la situation de soins). Cette 
transaction se veut réciproque: les actions de l'organisme influencent les conditions de l'environnement et les conditions de l'environnement influencent les actions de l'organisme. La transaction est aussi adaptative : l'organisme cherche à atteindre ses visées et s'adapte à l'environnement pour y parvenir.

Lorsqu'elle rencontre une situation de soins, l'infirmière observe attentivement ce qui se présente à elle, ce que Dewey (2007) nomme les « conditions environnementales ». Ceci permet de circonscrire la situation dans laquelle elle se trouve et d'identifier comment agir pour atteindre ses visées. La situation peut cependant devenir problématique dans trois circonstances : (1) si l'infirmière ne sait pas quelles actions mettre en place pour atteindre ses visées, (2) si les observations de l'infirmière ne correspondent pas à la conception qu'elle entretient d'un élément de la situation, et (3) lorsque les règles et principes que l'infirmière connait ne lui permettent pas d'expliquer ce qui se présente à elle.

Afin de résoudre une situation problématique, l'infirmière s'engagera dans une réflexion sur son expérience. La réflexion consiste en une démarche en cinq étapes (Dewey, 2007). Elle s'amorce par la survenue d'une situation problématique. S'en suit l'observation délibérée des conditions environnementales afin de définir le problème. Puis, par un mouvement inductif à partir des données d'observation, l'infirmière émet une ou des hypothèses pour expliquer la situation. Elle élabore l'hypothèse dans un mouvement déductif, en décrivant ses implications dans la situation. Ceci signifie qu'elle doit décrire ce qu'elle observerait si cette hypothèse était valide. Finalement, elle expérimente les actions en réactions à l'hypothèse, ce qui permet de comparer leurs implications anticipées avec leurs conséquences réelles. Un exemple visant à illustrer cette démarche est présentée dans l'encadré 1.

Encadré 1

Exemple d'une démarche de réflexion définie à partir des écrits de Dewey (2007, 1997)

\begin{tabular}{|c|c|}
\hline Mise en contexte & $\begin{array}{l}\text { Une infirmière prend soins d'un patient qui a subi une laparoscopie } \\
\text { abdominale. Malgré l'administration d'un analgésique, le patient } \\
\text { ressent une douleur importante à l'abdomen. }\end{array}$ \\
\hline $\begin{array}{l}\text { Situation } \\
\text { problématique }\end{array}$ & $\begin{array}{l}\text { Le patient se plaint quant à sa douleur abdominale. Cette situation } \\
\text { pourrait être problématique pour l'infirmière dans trois circonstances : } \\
\text { 1. L'infirmière ne connait pas d'autres moyen que la } \\
\text { médication pour soulager la douleur. } \\
\text { a. Elle ne connaît pas les actions à mettre en place } \\
\text { pour atteindre ses visées. } \\
\text { L. L'infirmière croit que la douleur suite à une laparoscopie } \\
\text { devrait être minimale. } \\
\text { a. Ce que l'infirmière observe dans la situation ne } \\
\text { correspond pas à sa conception de cette situation. } \\
\text { L'infirmière croit que l'administration d'un analgésique } \\
\text { s. } \quad \text { Ceulage toujours la douleur. } \\
\text { a. Ce règle ou ce principe connu de l'infirmière } \\
\text { ne semble pas s'appliquer dans cette situation. }\end{array}$ \\
\hline Observation délibérée & $\begin{array}{l}\text { L'infirmière procède à un recueil de données exhaustif en lien avec } \\
\text { la situation : description de la douleur, inspection du site chirurgical, } \\
\text { examen abdominal, signes vitaux, etc. Parmi les données recueillies, }\end{array}$ \\
\hline
\end{tabular}


l'infirmière note une distension et un tympanisme abdominaux.

\begin{tabular}{l|l}
\hline Émission d'hypothèses & $\begin{array}{c}\text { Pour expliquer la situation du patient, l'infirmière émet deux } \\
\text { hypothèses : }\end{array}$
\end{tabular}

1. Il peut s'agir d'une accumulation de sang dans l'abdomen.

2. Il peut s'agir d'une accumulation d'air dans l'abdomen.

Élaboration des

hypothèses
La première hypothèse, l'accumulation de sang, devrait provoquer une matité lors de la percussion abdominale. En effet, la percussion de structures denses (comme les liquides) provoque un son sourd de faible intensité. Or, l'infirmière a entendu un tympanisme, un son plus aigu et intense qui est associé à la percussion de structure contenant de l'air. Ainsi, les données cliniques ne permettent pas de conclure à l'accumulation de sang dans l'abdomen. Cette hypothèse est donc rejetée.

La seconde hypothèse, l'accumulation d'air, permettrait d'expliquer la distension abdominale et le tympanisme entendu lors de la percussion. L'infirmière sait aussi que durant une laparoscopie, de l'air est injecté au niveau de l'abdomen. La deuxième hypothèse apparait davantage plausible.

Si le patient présente une accumulation d'air dans l'abdomen et que cette accumulation d'air lui occasionne de la douleur, il est probable qu'il serait soulagé par l'évacuation de gaz.

Expérimentation

Apprentissage
Pour expérimenter la seconde hypothèse, l'infirmière met en place des interventions non-pharmacologiques pour stimuler le transit du patient et l'encourage à évacuer ses gaz. Suite à ces interventions, le patient se dit soulagé. L'hypothèse est donc validée : c'est bien une accumulation d'air au niveau abdominal qui provoquait la douleur. $\mathrm{Si}$ les interventions n'avaient pas atteint l'objectif souhaité, l'infirmière aurait dû poursuivre sa réflexion en émettant d'autres hypothèses.

Dans les semaines suivantes, l'infirmière prend soins d'un autre patient qui a subi une laparoscopie abdominale. Lorsque ce patient se plaint de douleur, l'infirmière examine immédiatement son abdomen et constate une distension et un tympanisme. Elle reconnaît alors la situation vécue précédemment et comprend que la douleur est liée à l'accumulation d'air dans l'abdomen du patient (meilleure différenciation de l'environnement). Elle met alors en place des interventions pour stimuler l'évacuation de gaz puisque ces interventions avaient été efficaces lors de la situation vécue précédemment (développement de mode de réponse).

La réflexion permet de comprendre une situation problématique et stimule des apprentissages de deux ordres. Premièrement, elle permet une meilleure différenciation de l'environnement. Ceci consiste à regrouper les données observées en un tout organisé et cohérent. Lorsque des données similaires se présenteront à nouveau, l'infirmière sera susceptible d'y porter attention et d'établir un lien avec la situation problématique qu'elle vient de rencontrer. Deuxièmement, l'infirmière qui juge que les actions qu'elle a mises en place lui ont 
permis d'atteindre ses visées sera portée à adopter ces modes de réponse pour le futur. Lorsque l'organisme différencie mieux son environnement et développe des modes de réponse appropriés, il a compris la signification d'une situation problématique. C'est ainsi que la réflexion peut être utilisée dans un contexte éducatif, lorsqu'un formateur invite un apprenant à une réflexion sur une expérience comme une opportunité d'apprentissage.

\section{Modèle pour la réflexion}

À partir de ces référents théoriques, un modèle de réflexion sur la pratique a été développé pour contribuer à l'apprentissage du raisonnement et du jugement cliniques infirmiers (figure 1; Lavoie, Pepin, \& Boyer, 2013). Ce modèle est appelé à être utilisé pour guider la réflexion sur une situation clinique réelle, auprès d'un patient, ou une situation clinique simulée, auprès d'un mannequin ou d'un acteur. Il s'agit, pour un formateur, d'accompagner l'apprenant dans sa réflexion sur cette situation selon une structure mettant l'accent sur le processus qui mène à un jugement clinique.

Figure 1

Modèle de réflexion pour le jugement clinique

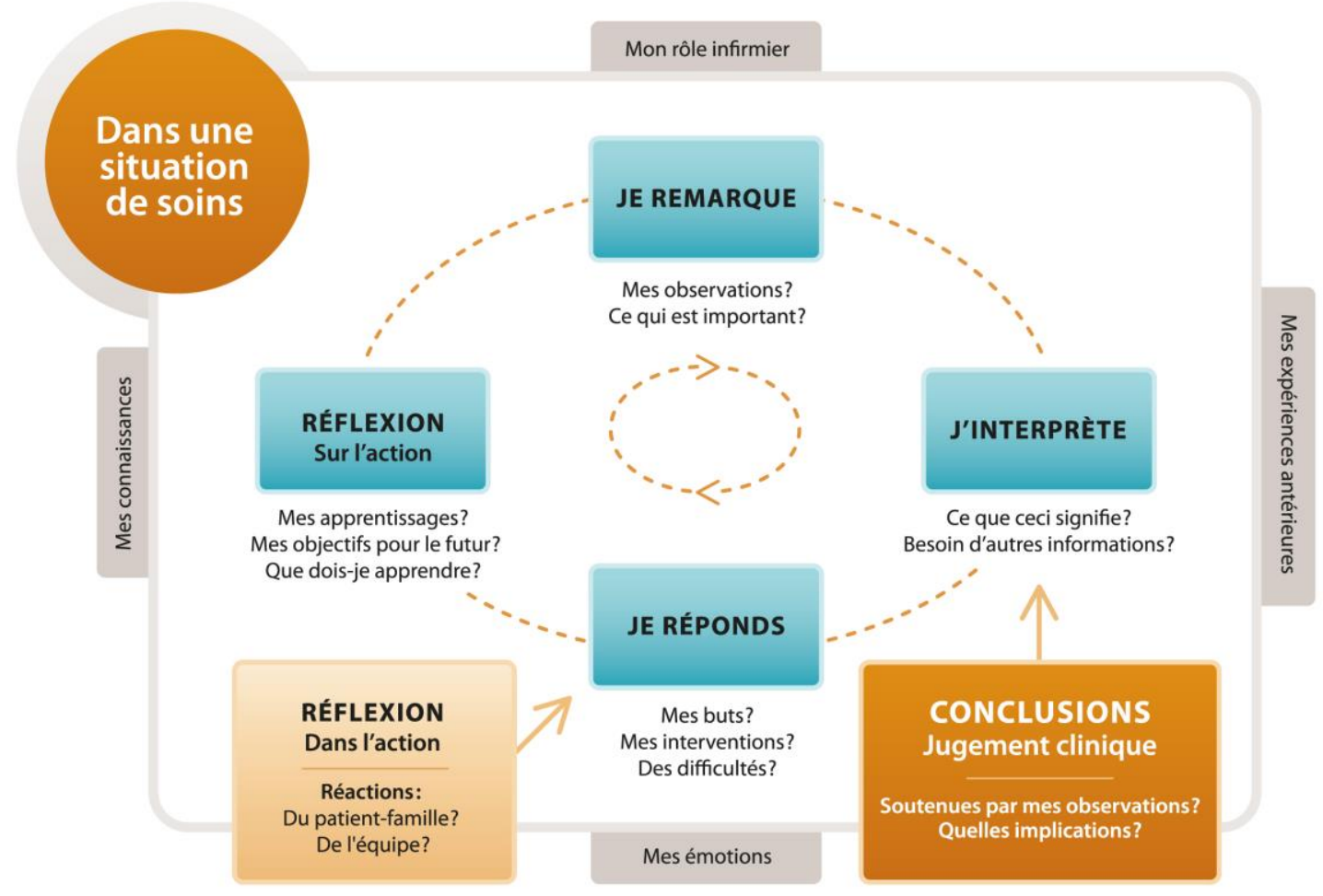

NOTE. Traduit et adapté de Lavoie et al. (2013)

Au centre du modèle se trouvent les étapes du processus de jugement clinique : je remarque, j'interprète, je réponds en réfléchissant dans l'action et je réfléchis sur l'action une fois qu'elle est complétée. Afin d'accompagner les apprenants dans la réflexion au moyen du modèle, le formateur utilise une approche de questionnement et des exemples de questions sont présentés. Ces questions pourront être adaptées en fonction de la situation clinique. Elles sont à la fois 
arrimées au processus de jugement clinique (Tanner, 2006), mais aussi à la démarche de réflexion telle que décrite par Dewey (2007).

D'abord, il est supposé que la situation sur laquelle les participants engagent une réflexion aura présenté un caractère problématique. Pour explorer ce que les apprenants y ont remarqué, le formateur les invitera à décrire leurs observations et à définir les données qui apparaissaient importantes dans la situation. À ce moment, il importera de dresser un portrait exhaustif de la situation en se gardant de tenter d'expliquer leur signification. Le formateur devra se livrer à l'exercice délicat de ramener les apprenants à la description des observations lorsque ceux-ci verseront dans l'explication. Il s'agira, par exemple, de décrire une hyperthermie sans tenter de l'expliquer par un processus infectieux.

C'est seulement lorsqu'un portrait exhaustif des données dans la situation aura été dressé que le formateur demandera de les interpréter. Ici, les apprenants seront invités à émettre des hypothèses par un mouvement inductif : comment attribuer du sens à un ensemble de données, qu'est-ce qu'elles signifient, qu'est-ce qui pourrait expliquer ce portrait? Le travail consistera à rassembler des données pour tenter de comprendre et d'expliquer la situation. Au besoin, des informations qui ont été omises lors de la description des observations pourront être ajoutées afin de compléter le portrait de la situation. Chaque hypothèse est accueillie ouvertement, sans être rejetée d'emblée.

Pour chaque hypothèse émise, le formateur demandera aux apprenants d'en décrire les implications afin de vérifier si les données recueillies soutiennent l'hypothèse. Dans un mouvement déductif, il amènera les apprenants à décrire les données qui seraient observées si l'hypothèse formulée s'avérait. Pour revenir à l'exemple du processus infectieux, les apprenants seraient amenés à décrire les implications de cette hypothèse : hyperthermie, tachycardie, augmentation des globules blancs, etc. Cet exercice leur permettra de comparer les implications de l'hypothèse avec ce qui s'est présenté dans la situation et de valider ou d'infirmer l'hypothèse. Ils en arriveront à rejeter les hypothèses formulées, si elles ne permettent pas d'expliquer les données observées, ou à identifier une ou des hypothèses plausibles.

Une fois qu'une hypothèse est jugée plausible puisqu'elle permet d'expliquer la majorité des observations, le formateur demandera aux apprenants de définir leur réponse face à cette hypothèse. Il s'agira de définir les buts visés, les interventions nécessaires et les difficultés potentielles. En explorant les réactions souhaitables face aux interventions, les apprenants pourront expérimenter différentes avenues et définir les résultats souhaités de leurs actions. Par exemple, le fait d'administrer un antipyrétique permettra de diminuer l'hyperthermie, sans toutefois entraver le processus infectieux, ce dernier nécessitant l'administration d'un antibiotique ou autre traitement pertinent. D'une part, ceci leur permettra de planifier leurs interventions en tenant compte des différentes cibles thérapeutiques; d'autre part, ceci vise à ce qu'ils puissent définir les effets souhaités de leurs interventions afin d'en évaluer l'efficacité.

En périphérie du modèle, les facteurs propres à l'infirmière qui peuvent influencer son jugement clinique sont représentés : la conception de l'infirmière de son rôle, ses expériences antérieures, les émotions qu'elle a ressenties pendant la situation et ses connaissances. À chaque étape de la réflexion, le formateur pourra inviter les apprenants à considérer comment ces facteurs ont pu exercer leur influence et comment ils peuvent nourrir la réflexion.

Il est à noter que la démarche de jugement clinique peut prendre une apparence linéaire dans ce modèle, alors qu'il s'agit d'un processus cyclique et itératif. En plus de faire remarquer ceci 
aux apprenants, le formateur expérimenté pourra effectuer des aller-retours entre les différentes étapes du modèle. Il est à noter que des recherches ont montré qu'avec l'expérience, le processus menant au jugement clinique tend à s'intégrer de plus en plus et à perdre de son aspect linéaire (Boyer et al., 2015). Il importe donc que le formateur soit sensible à l'expertise des apprenants et qu'il s'ajuste en conséquence.

Un exemple de mise en application du modèle de réflexion est présenté (encadré 2). Bien que l'exemple présenté corresponde à une situation de détérioration de l'état physique d'un patient, le modèle peut s'appliquer à d'autres sphères de soins. Aussi, il importe que le formateur ajuste la structure de la démarche réflexive en fonction du temps dont il dispose, de ses intentions pédagogiques et du niveau de développement des apprenants. En effet, la démarche réflexive pourrait être employée auprès d'étudiants en formation initiale, mais également auprès d'infirmières plus expérimentées dans le cadre d'activités régulières de formation continue.

Encadré 2

Exemple de mise en application du modèle de réflexion

Annie est une formatrice en soins infirmiers. Elle accompagne un groupe d'étudiants infirmiers de deuxième année de formation à l'occasion de leur stage en chirurgie générale. Lors de la rencontre de groupe suivant une journée de stage, Luc décrit la situation de santé de M. Gilles, un homme de 68 ans ayant subi une néphrectomie droite en raison d'une tumeur cancéreuse. Durant la journée, M. Gilles a présenté un saignement important au niveau du site opératoire qui a entrainé une altération de ses signes vitaux.

Puisque Luc exprime un certain inconfort (émotions) face à cette situation problématique, Annie décide d'entreprendre une démarche réflexive avec le groupe d'étudiants afin qu'ils apprennent de cette expérience. Elle les invite donc à considérer le diagramme du modèle de réflexion et amorce la discussion en posant des questions au groupe : "Luc, peux-tu nous raconter ce qui s'est passé aujourd'hui avec M. Gilles? » Une fois que Luc a présenté un portrait global de la situation clinique, Annie invite le groupe à amorcer la démarche réflexive.

1. Je remarque

Observation

délibérée des

conditions environnementales

Elle demande aux étudiants de décrire leur démarche de collecte de données auprès du patient. À cette occasion, l'utilisation d'un cadre de référence peut être utile afin de structurer l'évaluation du patient. Comme le stage s'inscrit dans un cours de chirurgie, Annie propose d'utiliser l'évaluation tête-pieds. Celle-ci consiste en une évaluation des paramètres physiques pertinents à la situation du patient, en débutant par la tête en en procédant successivement jusqu'aux pieds du patient. Ce faisant les étudiants décrivent les données d'évaluation relatives aux fonctions neurologique, respiratoire, cardiaque, abdominale et rénale du patient ainsi que l'ensemble de l'appareillage : oxygène, solutés, pansement au site chirurgical avec drain et sonde urinaire. Ils abordent également l'état psychologique du patient et la présence de sa conjointe à son chevet.

Une fois la collecte de données complétées, Annie invite les étudiants à identifier les données importantes: "Quelles sont les données qui vous tracassaient chez ce patient? Qu'est-ce qui ne correspond pas à ce que vous vous attendiez pour lui? ». Les étudiants 


\begin{tabular}{|c|c|}
\hline & $\begin{array}{l}\text { identifient différents problèmes : diminution de la pression artérielle, } \\
\text { tachycardie, accumulation de } 250 \mathrm{~mL} \text { de sang dans le drain en } 2 \text { heures, } \\
\text { diminution de la saturation périphérique en oxygène, anxiété du patient, } \\
\text { etc. }\end{array}$ \\
\hline $\begin{array}{l}\text { Inférence d'une } \\
\text { hypothèse } \\
\text { explicative } \\
\text { Élaboration des } \\
\text { hypothèses }\end{array}$ & $\begin{array}{l}\text { Une fois les données importantes identifiées, Annie demande : } \\
\text { «D'après vous, qu'est-ce qui pourrait expliquer ce que vous avez } \\
\text { observé chez ce patient? » Les étudiants identifient un ensemble } \\
\text { d'hypothèses, sans tenter de les valider : saignement au site chirurgical, } \\
\text { anxiété du patient, déshydratation, etc. Chaque hypothèse est accueillie } \\
\text { ouvertement, sans tenter de l'infirmer immédiatement. Il s'agit de } \\
\text { permettre aux apprenants d'exprimer toutes les idées qui leur sont } \\
\text { passées par la tête pour expliquer ce qui se présentait chez M. Gilles. } \\
\text { Annie reprend chacune des hypothèses et, dans un mouvement } \\
\text { déductif, demande aux étudiants si une hypothèse particulière } \\
\text { permettrait d'expliquer ce qui a été observé chez M. Gilles. Il s'agit de } \\
\text { vérifier si ces hypothèses permettent d'expliquer les observations des } \\
\text { étudiants. Par exemple, la déshydratation permettrait d'expliquer la } \\
\text { diminution de la pression artérielle, la tachycardie, la diminution de la } \\
\text { saturation et l'anxiété. Par contre, cette hypothèse ne permet pas } \\
\text { d'expliquer l'accumulation de sang dans le drain, contrairement au } \\
\text { saignement au site chirurgical qui permet d'expliquer tous les } \\
\text { problèmes recensés. Annie demande aux étudiants quelles seraient les } \\
\text { autres informations qui permettraient de valider cette hypothèse. Une } \\
\text { étudiante propose de regarder les résultats des prises de sang, afin de } \\
\text { vérifier les valeurs d'hémoglobine et d'hématocrite. Aussi, malgré qu'il } \\
\text { s'agisse d'un signe tardif de diminution du volume circulatoire, un } \\
\text { autre étudiant propose que la diurèse ait pu être diminuée. L'étudiant } \\
\text { avait observé ceci lors d'une situation similaire qu'il avait vécu auprès } \\
\text { d'un autre patient, dans le passé (expériences antérieures). }\end{array}$ \\
\hline 3. Je réponds & $\begin{array}{l}\text { Une fois l'hypothèse du saignement choisie par le groupe comme } \\
\text { étant la plus plausible, Annie propose de l'expérimenter. Puisqu'il n'y a } \\
\text { pas de patient qui présente actuellement une hémorragie, elle propose } \\
\text { une expérimentation théorique : «S'il s'agissait bien d'un saignement, } \\
\text { quelles seraient vos buts auprès de M. Gilles? Quelles auraient été les } \\
\text { interventions pertinentes et quels seraient les résultats attendus? » Les } \\
\text { étudiants expliquent que leurs buts auraient été de limiter le saignement } \\
\text { et d'assurer une perfusion systémique adéquate, malgré la diminution } \\
\text { du volume sanguin. Puisque le saignement était seulement visible au } \\
\text { niveau du drain, il s'agissait probablement d'un saignement interne au } \\
\text { site opératoire. Ainsi, il devenait nécessaire d'obtenir l'assistance du } \\
\text { chirurgien. Aussi, les étudiants proposent que l'administration de } \\
\text { liquide intraveineux en quantité importante ait pu remplacer le volume } \\
\text { sanguin perdu. L'efficacité de cette intervention aurait été observable } \\
\text { par l'augmentation de la pression artérielle et une normalisation de la } \\
\text { fréquence cardiaque. Pour ce qui est de la saturation en oxygène, ils } \\
\text { proposent d'augmenter le débit d'oxygène le temps de rétablir le }\end{array}$ \\
\hline
\end{tabular}


volume circulant et d'assurer un suivi afin de ne pas en administrer inutilement. Ils proposent également de baisser la tête du lit du patient afin d'assurer une meilleure répartition du volume sanguin, tout en s'assurant de ne pas compromettre sa fonction respiratoire. Le groupe aborde l'importance d'effectuer des interventions de soutien auprès de M. Gilles et de sa conjointe afin de diminuer l'anxiété du patient et de les accompagner dans cette expérience difficile. Le groupe en profite pour se questionner sur les interventions à effectuer de manière autonome et celles nécessitant l'avis d'un médecin (rôle infirmier). En explicitant les effets attendus de chaque intervention, Annie amène les étudiants vers la «réflexion-dans-l'action ». C'est qu'elle invite les étudiants à se questionner sur les réactions attendues du patient, de sa famille et de l'équipe de soins face à ces interventions. Elle incite également les étudiants à réfléchir à des difficultés potentielles dans la mise en œuvre de leur plan d'action.

4. Réflexion sur Au terme de la démarche réflexive, Annie invite les étudiants à l'action décrire un apprentissage réalisé au cours de la discussion. Elle se situe maintenant à l'étape «réflexion sur l'action». Pour chaque apprentissage, l'étudiant est invité à le transformer en objectif. Par exemple, Luc explique qu'il a compris l'importance de procéder à une évaluation systématique des patients, même lors des situations où leur état se détériore. Cet apprentissage est converti en objectif : procéder à une évaluation tête-pied pour chaque patient lors de la prochaine journée de stage. Par le fait même, Luc exprime son inconfort face aux techniques d'évaluation respiratoire et se fixe l'objectif de réviser la théorie et les procédures d'évaluation liées à ce système (connaissances).

NOTE. Les éléments en italique et entre parenthèses constituent les facteurs d'influence du jugement clinique qui sont présentés en périphérie de la Figure 1.

\section{Des résultats de recherche en lien avec le modèle}

\section{Après une simulation clinique}

Une première version du modèle de réflexion a été développée pour être déployée auprès d'infirmières novices sur une unité de soins critiques (Lavoie et al., 2013). Il s'agissait alors d'un modèle qui visait à structurer le débriefing post-simulation clinique où un patient ayant subi une neurochirurgie présentait une arythmie cardiaque avec altération hémodynamique. Les cinq infirmières ayant participé à cet essai pilote ont complété un questionnaire sur leur satisfaction et leur perception de l'utilité du débriefing selon ce modèle de réflexion. Les résultats montrent qu'elles ont perçu qu'il leur avait permis de mieux comprendre la situation de soins et comment elles étaient arrivées à prendre certaines décisions dans la simulation. Elles ont également perçu que cet exercice pouvait contribuer à améliorer la priorisation et l'organisation de leurs soins, leurs habiletés d'évaluation clinique et leur jugement clinique dans une situation similaire.

Plus récemment, le travail de développement du modèle a été poursuivi pour qu'il soit adapté au débriefing d'un scénario de simulation clinique où un patient présente une détérioration de son état de santé (Lavoie, Pepin, \& Cossette, 2015). Cette version du modèle de 
réflexion met notamment de l'avant une structure d'évaluation de la santé propre aux situations de soins critiques, soit l'évaluation $\mathrm{ABCDE}^{1}$ (Emergency Nurses Association, 2007). Pour évaluer la contribution du débriefing réflexif au jugement clinique, une expérimentation classique a été mise en œuvre auprès de 119 étudiants infirmiers qui participaient à des simulations de détérioration du patient. En comparaison avec un retour évaluatif de type PlusDelta $^{2}$ (Fanning \& Gaba, 2007), les étudiants qui ont participé à des retours réflexifs selon le modèle ont connu une plus grande amélioration de leurs observations des données cliniques dans la simulation. C'est donc dire que l'approche réflexive leur a permis d'améliorer leur capacité d'évaluation et de collecte de données auprès du patient. Un effet positif a également été observé sur leur compréhension de la situation. Cet effet était comparable à celui du Plus-Delta. Lorsque les étudiants ont été questionnés sur l'apport de l'approche réflexive à leurs apprentissages, ils ont révélé des facteurs qui pourraient expliquer les effets observés. Parmi ceux-ci, la revue des données cliniques dans la simulation par une approche systématique ABCDE les aurait aidés à apprendre. Le fait de discuter et de réfléchir sur une situation de soins en petit groupe de pairs, tout en étant guidé par les questions du formateur, aurait été un autre facteur positif (Lavoie, Pepin, \& Cossette, 2017).

\section{Pour des activités de formation continue}

Le modèle a également été adapté pour être mis à l'essai lors de séances de réflexion en petit groupe, sur des unités de soins $(n=8)$ de deux centres hospitaliers universitaires québécois (Goudreau et al., 2015). Ces séances regroupaient des infirmières d'une même équipe, avec l'intention de soutenir le développement du raisonnement et du jugement cliniques des infirmières nouvellement diplômées qui intégraient ces équipes. Elles réfléchissaient ensemble à des situations cliniques vécues par l'équipe pendant une trentaine de minutes. Les situations abordées lors de ces rencontres ont grandement varié : de la détérioration d'un patient précédant un arrêt cardiorespiratoire à l'adaptation des soins en fonction de la culture du patient. Les infirmières nouvellement diplômées ayant participé à l'étude $(n=55)$ ont révélé que leur participation aux séances de réflexion leur avait permis de faire plus de liens entre les données recueillies lors de l'évaluation des patient et d'identifier les données supplémentaires devant être recueillies. Le fait de partager avec les collègues sur des situations problématiques leur a permis d'imaginer collectivement de nouvelles avenues d'interventions possibles. Participer aux séances de réflexion leur aurait également permis d'améliorer leur confiance en elles et de mieux comprendre leur rôle au sein d'une équipe interprofessionnelle. Ceci fait en sorte que le modèle de réflexion pourrait également avoir contribué au développement de leur leadership clinique infirmier.

Au terme de cette étude, des groupes de participants de deux unités de soins ont décidé de poursuivre les séances de réflexion. Lorsque questionnés à cet effet, ces participants ont affirmé qu'une compréhension approfondie de la démarche réflexive et une conviction profonde quant aux effets bénéfiques des séances régulières de réflexion constituaient des facteurs ayant contribué à leur volonté de poursuivre au-delà de l'étude. Aussi, ces participants bénéficiaient d'un soutien important de leur chef d'unité.

\footnotetext{
${ }^{1}$ Airway (perméabilité des voies respiratoires); Breathing (évaluation de la respiration); Circulation (évaluation de l'état circulatoire); neurological Disability (évaluation de l'état neurologique); Environment (évaluation des conditions environnementales).

${ }^{2}$ Le Plus-Delta consiste à demander aux étudiants ce qui a bien été (plus), ce qui devrait être amélioré (delta) et comment ceci pourrait être amélioré pour une prochaine fois.
} 


\section{Discussion}

Cet article présente un modèle pour accompagner les infirmières et les étudiants dans leur réflexion sur des situations de soins afin qu'elles développent leur raisonnement et leur jugement clinique. Ce modèle se fonde sur l'arrimage d'un modèle de jugement clinique et d'une théorie de la réflexion et de l'apprentissage expérientiel. Les études présentées montrent que sa mise en œuvre dans différentes stratégies pédagogiques actives était faisable, acceptable et présentait des bénéfices pour l'apprentissage. Il est important de noter que ce modèle s'adresse à des infirmières étudiantes ou novices et que des infirmières plus expérimentées font ce cheminement dans leur tête, sans nécessairement l'expliciter (Benner, 1984; Benner et al., 2009; Tanner, 2006). Il pourra néanmoins constituer un atout pour assister les plus expertes dans la verbalisation de leur processus de raisonnement et de jugement clinique, notamment pour accompagner les débutantes. Il est à souligner que dans l'étude de Goudreau et al. (2015), la participation conjointe d'infirmières novices et plus expérimentées aux séances de réflexion en petit groupe a été un des facteurs de succès de l'activité.

Il est souhaitable que ce modèle continue d'être mis en œuvre dans différents contextes d'apprentissage actif. Les formateurs sont invités à adapter ses éléments pour qu'ils s'arriment étroitement aux particularités des situations cliniques rencontrées. Les éléments du modèle qui pourraient être modifiés comprennent notamment la manière de décrire les observations dans la situation. Par exemple, il serait à propos, dans le cas d'un patient vivant une situation de santé mentale, de décrire sa situation en adoptant une démarche d'évaluation spécifique à ce domaine (Cloutier \& Leclerc, 2011). Pour ce qui est des autres éléments du modèle, l'expertise clinique des formateurs devrait les soutenir dans la définition des hypothèses pertinentes ainsi que des buts et des résultats attendus des interventions. Dans une certaine mesure, il s'agit de rendre visible le processus de raisonnement et de jugement clinique de l'apprenant afin d'accompagner son raffinement grâce à l'expertise du formateur. Il s'agit également d'une occasion de définir des cibles d'apprentissage pour parfaire les connaissances, les habiletés et les attitudes de l'apprenant dans une optique de développement de compétences.

Pour ce qui est de l'évaluation de la mise en œuvre du modèle, il est possible d'utiliser des indicateurs de satisfaction des apprenants qui témoigneront de sa faisabilité et de son acceptabilité. Quant à ses bénéfices, l'observation des actions des apprenants à même des situations cliniques réelles ou simulées offre le potentiel de constater les apprentissages réalisés. À cette fin, des rubriques ou des grilles avec critères ont été élaborées et comprennent une série d'indicateurs qui permettent d'apprécier la qualité du raisonnement et du jugement clinique des infirmières et des étudiantes (Shipman, Roa, Hooten, \& Wang, 2012). Elles peuvent s'utiliser dans une situation clinique précise et isolée dans le temps (Lasater, 2007; Radhakrishnan, Roche, \& Cunningham, 2007) ou encore dans une perspective longitudinale du développement de ces compétences (Boyer et al., 2015). Les référentiels de compétences sont d'autres outils qui peuvent servir à témoigner de l'apprentissage des apprenants.

Il importe néanmoins de spécifier que le succès dans l'utilisation du modèle dépend à la fois de l'engagement des apprenants et des formateurs dans son utilisation. Lors de son implantation en milieu clinique (Goudreau et al., 2015), les infirmières des équipes ne se sentaient pas complètement à l'aise pour animer les premières séances de réflexion. Un soutien important de la part des gestionnaires a été nécessaires à la fois pour planifier du temps pour les séances, mais aussi pour que les animatrices s'approprient l'utilisation du modèle de réflexion. Qui plus est, il a été noté que la motivation des participants et des formateurs était un facteur important de succès 
et de pérennité des séances de réflexion, ce qui a aussi été observé dans d'autres études (Bailey \& Graham, 2007; Kenny \& Allenby, 2013). Cette motivation s'observait par leur reconnaissance explicite de la valeur ajoutée des séances de réflexion et par leur volonté de réserver du temps à cet effet.

Par ailleurs, les résultats des études précédentes montrent que le fait de revisiter des situations vécues comme problématiques a été une source d'émotions négatives pour certains participants, ce qui a pu nuire à leur apprentissage (Lavoie et al., 2013; Goudreau et al., 2015). Il a été perçu que la nature même des évènements abordés pouvait influencer positivement ou négativement le climat des activités de réflexion. L'établissement d'un environnement d'ouverture, de respect et de confiance est donc à prioriser pour une utilisation efficace de cet outil de même que pour toute activité de formation qui mise sur la réflexion (Dawber, 2013; McVey \& Jones, 2012; Olofsson, 2005).

\section{Conclusion}

Le modèle de réflexion proposé se veut un outil d'accompagnement auprès d'infirmières et d'étudiantes qui rencontrent des situations cliniques pouvant être des occasions d'apprentissage actif. Combiné à l'expertise clinique des formateurs en soins infirmiers, ce modèle constitue un moyen de contribuer au raisonnement et au jugement clinique des apprenants. À ce jour, il a été utilisé dans le contexte de la simulation clinique et de la formation continue d'infirmières en pratique clinique. De futurs travaux seront à mener pour obtenir des résultats de recherche quant à sa mise à l'essai dans le contexte des stages en soins infirmiers. 


\section{Références}

Alligood, M. R. (2013). Nursing theory: Utilization \& application (5th ed.). Maryland Heights, MO: Elsevier Health Sciences.

Bailey, M. E., et Graham, M. M. (2007). Introducing guided group reflective practice in an Irish palliative care unit. International Journal of Palliative Nursing, 13, 555-560. doi:10.12968/ijpn.2007.13.11.27591

Banning, M. (2008). A review of clinical decision making: Models and current research. Journal of Clinical Nursing, 17, 187-195. doi:10.1111/j.1365-2702.2006.01791.x

Benner, P. (1984). From novice to expert: Excellence and power in clinical practice. Menlo Park, CA: Addison-Wesley.

Benner, P., Tanner, C., et Chesla, C. (2009). Expertise in nursing practice: Caring, judgment, and ethics (2nd ed.). New York, NY: Springer.

Bonwell, C. C., et Eison, J. A. (1991). Active learning: Creating excitement in the classroom. Washington, DC: The George Washington University, School of Education and Human Development.

Boyer, L., Tardif, J., et Lefebvre, H. (2015). From a medical problem to a health experience: How nursing students think in clinical situations. Journal of Nursing Education, 54, 625632. doi:10.3928/01484834-20151016-03

Cloutier, L., et Leclerc, C. (2011). L'évaluation de la condition mentale. Perspective infirmière, $8(3), 29-31$.

Dawber, C. (2013). Reflective practice groups for nurses: A consultation liaison psychiatry nursing initiative: Part 2-The evaluation. International Journal of Mental Health Nursing, 22, 241-248. doi:10.1111/j.1447-0349.2012.00841.x

Dewey, J. (1997). Experience and education. New York, NY: Touchstone.

Dewey, J. (2007). How we think. Stilwel, KS: Digireads.com.

Emergency Nurses Association. (2007). Trauma nursing core course (TNCC) provider manual. (6th ed.). Des Plaines, IL: Emergency Nurses Association.

Fanning, R. M., et Gaba, D. M. (2007). The role of debriefing in simulation-based learning. Simulation in Healthcare, 2, 115-125. doi:10.1097/SIH.0b013e3180315539

Frank, J. R., Mungroo, R., Ahmad, Y., Wang, M., De Rossi, S., et Horsley, T. (2010). Toward a definition of competency-based education in medicine: a systematic review of published definitions. Medical Teacher, 32, 631-637. doi:10.3109/0142159X.2010.500898

Fonteyn, M. E. (1998). Thinking strategies for nursing practice. Philadelphie, PA : Lippincott Williams \& Wilkins.

Goudreau, J., Pepin, J., Dubois, S., Boyer, L., Larue, C., et Legault, A. (2009). A second generation of the competency-based approach to nursing education. International Journal of Nursing Education Scholarship, 6, Article 15. doi:10.2202/1548-923x.1685

Goudreau, J., Pepin, J., Larue, C., Dubois, S., Descoteaux, R., Lavoie, P., et Dumont, K. (2015). A competency-based approach to nurses' continuing education for clinical reasoning and 
leadership through reflective practice in a care situation. Nurse Education in Practice, 15, 572-578. doi:10.1016/j.nepr.2015.10.013

Higgs, J. (2008). Clinical reasoning in the health professions. Boston, MA: Elsevier Health Sciences.

Kenny, A., et Allenby, A., (2013). Implementing clinical supervision for Australian rural nurses. Nurse Education in Practice, 13, 165-169. doi:10.1016/j.nepr.2012.08.009

Lasater, K. (2007). Clinical judgment development: Using simulation to create an assessment rubric. Journal of Nursing Education, 46, 496-503.

Lavoie, P., Pepin, J., et Boyer, L. (2013). Reflective debriefing to promote novice nurses' clinical judgment after high-fidelity clinical simulation: A pilot test. Dynamics, 24(4), 36-41.

Lavoie, P., Pepin, J., et Cossette, S. (2015). Development of a post-simulation debriefing intervention to prepare nurses and nursing students to care for deteriorating patients. Nurse Education in Practice, 15, 181-191. doi:10.1016/j.nepr.2015.01.006

Lavoie, P., Pepin, J., et Cossette, S. (2017). Contribution of a reflective debriefing to nursing students' clinical judgment in patient deterioration simulations: A mixed-methods study. Nurse Education Today, 50, 51-56. doi:10.1016/j.nedt.2016.12.002

McVey, J., et Jones, T. (2012). Assessing the value of facilitated reflective practice groups. Cancer Nursing Practice, 11(8), 32-37. doi:https://doi.org/10.7748/cnp2012.10.11.8.32.c9357

Olofsson, B. (2005). Opening up: Psychiatric nurses' experiences of participating in reflection groups focusing on the use of coercion. Journal of Psychiatric and Mental Health Nursing, 12, 259-267. doi:10.1111/j.1365-2850.2005.00827.x

Prince, M. (2004). Does active learning work? A review of the research. Journal of Engineering Education, 93, 223-231. doi:10.1002/j.2168-9830.2004.tb00809.x

Radhakrishnan, K., Roche, J., et Cunningham, H. (2007). Measuring clinical practice parameters with human patient simulation: A pilot study. International Journal of Nursing Education Scholarship, 4, Article 8. doi:10.2202/1548-923X.1307

Shipman, D., Roa, M., Hooten, J., et Wang, Z. J. (2012). Using the analytic rubric as an evaluation tool in nursing education: The positive and the negative. Nurse Education Today, 32, 246-9. doi:10.1016/j.nedt.2011.04.007

Tanner, C. A. (2006). Thinking like a nurse: A research-based model of clinical judgment in nursing. Journal of Nursing Education, 45, 204-11. 